\title{
Optical turbulence modeling in the boundary layer and free atmosphere using instrumented meteorological balloons
}

\author{
A. Abahamid ${ }^{1}$, A. Jabiri ${ }^{1}$, J. Vernin ${ }^{2}$, Z. Benkhaldoun ${ }^{1}$, M. Azouit ${ }^{2}$, and A. Agabi ${ }^{2}$ \\ ${ }^{1}$ Laboratoire de Physique des Hautes Énergies et Astrophysique, Département de Physique, \\ Faculté des Sciences Semlalia, BP 2390, Marrakech, Maroc \\ 2 LUAN, UMR 6525, Université de Nice-Sophia Antipolis, 06108 Nice Cedex 2, France
}

Received 12 May 2003 / Accepted 25 August 2003

\begin{abstract}
In this paper, we study the behavior of atmospheric turbulence in the boundary layer and free atmosphere using balloon-born radiosondes. The data measurements were carried out with 168 balloons launched from 9 sites. Profiles of various parameters $\left(C_{N}^{2}, M^{2}\right.$ and $\left.L_{\mathrm{o}}\right)$ characterizing atmospheric turbulence are established. We work out statistical models of atmospheric turbulence in the boundary layer, and we checked their coherence by comparing them with other models. In the free atmosphere, the $C_{N}^{2}$ profile is coherent with those of the models of Hufnagel and of Brown-Beland. A complete behavior of the outer scale $L_{\mathrm{o}}$ is given from the ground up to $30 \mathrm{~km}$ which fits with partial models of Coulman-Vernin and Beland-Brown.
\end{abstract}

Key words. turbulence - atmospheric effects - balloons

\section{Introduction}

The performances of large telescopes are highly dependent upon optical turbulence. The spatial distribution of the optical turbulence depends on meteorological conditions (wind, temperature, pressure and humidity) and also on local orographic disturbances. These parameters influence mainly the refractive index inhomogeneities throughout the atmosphere. Therefore, the knowledge of the spatial statistic of refractive index fluctuations is essential in the study of wave propagation in the atmosphere.

Optical turbulence modeling in the boundary layer allows us to forecast the observation conditions. Experiments have to verify some theoretical laws and to provide expressions of stability functions. Turbulence in the surface layer has been studied by Wyngaard et al. (1971), for example, with the Kansas experiment. From these meteorological measurements, Wyngaard proposed similitude laws for the structure coefficient of the refractive index $C_{N}^{2}$ that allows one to find asymptotic behavior of the $C_{N}^{2}$ profiles versus height.

The progress in meteorological simulations has been used to characterize wave propagation in a turbulent medium. The use of balloon-born thermal probes initiated by Coulman (1973), and Bufton (1973a,b) greatly improved our knowledge. These in situ experiments are very useful tools for investigating astronomical seeing without using large telescopes. The same technique has been extensively used by Barletti et al. (1974, 1977) during the J.O.S.O. site testing campaign. In 1988, Coulman established a numerical model for the outer

Send offprint requests to: A. Jabiri, e-mail: jean@vernin@unice.fr scale of turbulence using meteorological radiosonde data and Scidar measurements. Since this time, the turbulence modeling experienced a considerable development e.g. Beland \& Brown (1988), Bougeault (1995), and Masciadri (1998).

In this paper, we present a statistical study of various parameters $\left(C_{N}^{2}, M^{2}\right.$ and $\left.L_{\mathrm{o}}\right)$, which provides useful information relevant to the physics of turbulence within the boundary layer and the free atmosphere.

\section{Data processing}

\subsection{Theory}

The theory of light propagation through a turbulent medium is now well established (Tatarski 1961; Roddier 1981). The influence of atmospheric turbulence on the degradation of optical images has been widely investigated by several authors. Semi-empirical atmospheric models of turbulence intensity in the atmosphere with height are also given by Fried (1966) and Hufnagel (1974).

The traditional way to characterize image degradation in astronomy is to measure the "seeing". This parameter gives a measure of the optical turbulence intensity related to the refractive index inhomogeneities. The seeing is described in terms of the full width at half-maximum intensity $\varepsilon_{F W H M}$ of a star at the focus of a telescope:

$\varepsilon_{F W H M}=5.25 \lambda^{-1 / 5}\left(\int_{0}^{\infty} C_{N}^{2}(h) \mathrm{d} h\right)^{\frac{3}{5}}$ 
where $\lambda$ is the optical wavelength and $C_{N}^{2}(h)$, the structure coefficient of the refractive index, is given by Tatarski (1961):

$C_{N}^{2} \approx 2.8 M^{2} L_{\mathrm{o}}^{\frac{4}{3}}$.

$L_{\mathrm{o}}$ is the outer scale of optical turbulence (expressed in m) defined in Kolmogorov theory and $M$ the vertical gradient of potential refractive index, given by Coulman (1988):

$M=\frac{\delta N}{\delta h}=-\left(\frac{A P}{T}\right) \frac{1}{\theta} \frac{\delta \theta}{\delta h}$

$M$ is expressed in $\mathrm{m}^{-1}$ and $A=80 \times 10^{-6} \mathrm{~K} \mathrm{hPa}^{-1}$.

$P$ is the pressure expressed in $\mathrm{hPa}, T$ is the absolute temperature (in $\mathrm{K}$ ) and $\theta$ is the potential temperature (in K) defined by:

$\theta=T\left(\frac{1000}{P}\right)^{0.286}$.

Now, it is well established (Vernin \& Muñoz-Tuñón 1992) that, in order to characterize a site for astronomical observation, it is necessary to know the turbulence in the boundary layer and in the free atmosphere. Therefore it is necessary to probe the atmosphere and to measure vertical profiles of turbulence to test or select a site for astronomical observations.

\subsection{Physical separation between boundary layer and free atmosphere}

Various physical factors involved in the generation of atmospheric turbulence lead us to separate the atmosphere into two slabs, the boundary layer and the free atmosphere.

\subsubsection{Boundary layer}

The boundary layer is the lower part of the atmosphere where thermodynamic properties are directly influenced by the interaction with the ground. This layer is dominated by daily variations of weather conditions. Measurements carried out in various sites showed that the atmospheric boundary layer could, according to the site, contribute to a major part of the whole atmospheric turbulence (i.e. ESO-VLT report, 1987 No. 55, from Sarazin). In few other sites, like at Mauna Kea, Hawaii (Roddier 1990) or La Palma in the Canary Islands (Vernin \& Muñoz-Tuñon 1992), the boundary layer and the free atmosphere share an equal contribution to the overall optical turbulence. The boundary layer extends from the ground up to an altitude which varies from about 200 to $2000 \mathrm{~m}$, depending on diurnal and weather conditions. In this paper, where only night measurements are processed, we checked that $1 \mathrm{~km}$ is a relevant threshold for model validity. Since our profiles were taken from various sites at various ground altitudes, our boundary layer (BL) processing is performed taking into account the above ground altitude, from 0 to $1 \mathrm{~km}$.

\subsubsection{Free atmosphere}

The free atmosphere is the higher part of the atmosphere generally influenced by longer-term and meso-scale meteorological variations. It extends from the first kilometer above ground until the limit of the Earth's atmosphere (i.e. towards $30 \mathrm{~km}$, in this study). It is the part of the atmosphere in which the ground effects influence no longeron horizontal flow, and we took sea level as the altitude reference. In other words, our free atmosphere processing refers to ASL altitudes, excluding the first kilometer from ground level which is used in the BL analysis. Low ASL altitudes allow a poorer statistical accuracy than at higher levels, where all the flights pass through.

\subsubsection{Vertical resolution $\Delta h$}

Since the vertical ascent speed of the balloon is about $4 \mathrm{~m} \mathrm{~s}^{-1}$ and the temporal sampling is about $1.5 \mathrm{~s}$, the vertical resolution is about $6 \mathrm{~m}$. We processed our data within slabs of various thickness $(\Delta h=25,50,75$, and $100 \mathrm{~m})$ to reduce the noise which appears mainly in Eq. (3).

Inside a $\Delta h$ layer, and for flight $j$ we sort the $h_{i}$ altitudes where measurements exist either to compute $<C_{N}^{2}>\left.\right|_{\Delta h, j}$ or to fit a least square line to assess $\left.\langle M\rangle\right|_{\Delta h, j}$, the mean refractive index gradient, and thus the outer scale $\left.L_{\mathrm{o}}\right|_{\Delta h, j}$.

\subsection{Measurement technique}

The measurement method involves the launching of meteorological balloons equipped with sensors that assess the microstructure of the thermal field during their free flight ascent the ground level up to approximately $30 \mathrm{~km}$. A technique similar to that of Barletti et al. (1977) is used for the temperature structure function, which is defined as:

$D_{T}(\vec{r})=\left\langle|T(\vec{x})-T(\vec{x}+\vec{r})|^{2}\right\rangle$

and is assessed by means of two $5 \mu \mathrm{m}$ Wolfram wires separated by a distance $r$.

The temperature and the refractive index, $T$ and $N$ respectively, assumed to be passive and conservative additives, follow the Kolmogorov spectral law, leading to:

$D_{T}(r, h)=C_{T}^{2}(h) r^{\frac{2}{3}}$

where $l_{\mathrm{o}}<r<L_{\mathrm{o}}, l_{\mathrm{o}}$ and $L_{\mathrm{o}}$ are the inner and the outer scale of the optical turbulence. With our balloons, $r$ can be chosen between a few $\mathrm{cm}$ and few $\mathrm{m}$. In this paper, all separations are $r=1 \mathrm{~m}$. The structure coefficient of the refractive index $C_{N}^{2}(h)$ can be easily obtained from $C_{T}^{2}(h)$ using:

$C_{N}^{2}(h)=\left(80 \times 10^{-6} \frac{P(h)}{T^{2}(h)}\right)^{2} C_{T}^{2}(h)$.

The payload, developed at the Département d'Astrophysique of Nice University (Vernin \& Muñoz-Tuñón 1992), consists of an electronic board to compute $C_{T}^{2}(h)$ coupled to an "off the shelf" classical Vaissala meteorological sonde (to measure, $P, T, \mathrm{Rh}$ and wind) lifted to $30 \mathrm{~km}$ by a balloon at a vertical velocity of about $4 \mathrm{~m} \mathrm{~s}^{-1}$. The radiosonde is attached to the balloon by a long $(\sim 50 \mathrm{~m})$ cord in order to avoid any possible turbulent wake effects.

The number and sites where the flights took place are listed in Table 1. 


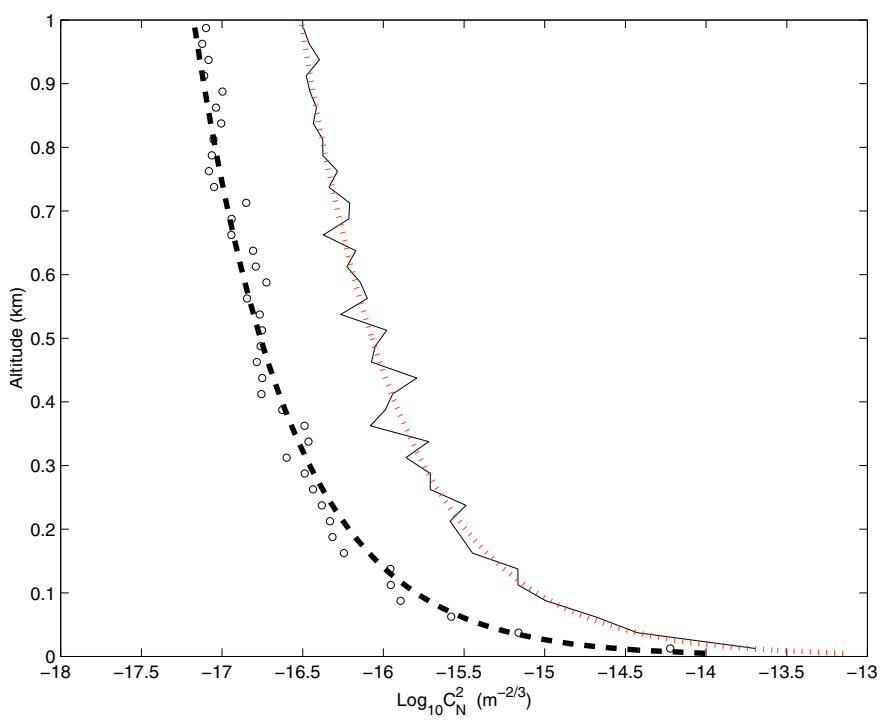

Fig. 1. $C_{N}^{2}$ vertical profile from sounding data computed for $\Delta h=25 \mathrm{~m}$ in the boundary layer. o: median; solid line: mean; dotted line and dotted-fat line: functions obtained by performing a least squares exponential fit.

\section{Results and discussion}

According to Eq. (2) the outer scale $L_{\mathrm{o}}$ can take very large values if $M \sim 0$, i.e. when the potential vertical gradient $\frac{\delta \theta}{\delta h} \sim 0$. In order to avoid too large $L_{\mathrm{o}}$ values we discarded the largest ones, which correspond to $M^{2} \leq 10^{-21}$, leading to a loss of about $3 \%$ of the processed data.

\subsection{Boundary layer}

In Figs. 1 to 6 are shown mean/median vertical profiles of $C_{N}^{2}$, $M^{2}$ and $L_{\mathrm{o}}$ in the boundary layer for various vertical resolution $\Delta h$. One can see that the three parameters behave like decreasing functions with altitude which can be fitted with an exponential law in the following way:

$Y(h)=A h^{-p}$,

$Y$ being $C_{N}^{2}, M^{2}$ or $L_{\mathrm{o}}$, with different $A$ and $p$.

Each experimental data set of $C_{N}^{2}, M^{2}$ and $L_{\mathrm{o}}$, average and median, were "least squares" fitted with an exponential law. The functions which best correlate with the median and mean data are presented in Tables 2-10 for each layers thickness $\Delta h$. The corresponding standard error is defined by:

$\sigma_{\mathrm{e}}=\left[\frac{1}{N-2} \sum\left(\log \left(Y_{i}^{\prime}\right)-\log \left(Y_{i}\right)\right)^{2}\right]^{(1 / 2)}$

where $Y_{i}^{\prime}$ is the median experimental values (mean values respectively) of $C_{N}^{2}\left(M^{2}\right.$ and $L_{\mathrm{o}}$ respectively) and $Y_{i}=A h_{i}^{-p}$ is the fitting function.

\section{- Structure constant of refractive index $C_{N}^{2}$ :}

In Fig. 1, we present the vertical profiles of the median and mean $C_{N}^{2}$ in the boundary layer, with only the $\Delta h=25 \mathrm{~m}$ results, for clarity. In Fig. 2 we show the same profiles for different layer thickness $\Delta h$. The values rise abruptly when approaching the first meters corresponding to the surface layer.
Table 1. Sites, altitudes and number of the balloon flights reported herein.

\begin{tabular}{ccc}
\hline \hline Site & Alt. (m) & Number of flights \\
\hline & 2400 & 6 \\
La Palma & 2200 & 8 \\
& 2150 & 7 \\
\hline O.H.P & 650 & 34 \\
Sirene & 1100 & 19 \\
Cerro Paranal & 2500 & 16 \\
South Pole & 2835 & 18 \\
Cerro Pachon & 2715 & 45 \\
Toulouse & 153 & 1 \\
San Pedro Mártir & 2440 & 12 \\
Aire sur l'Adour & 57 & 2 \\
\hline total & & 168 \\
\hline
\end{tabular}

Table 2. Fitting parameters $A$ and $p$, for various vertical resolutions, corresponding to $C_{N_{\text {median }}}^{2}$ values in the boundary layer.

\begin{tabular}{cccc}
\hline \hline$\Delta h$ & $A$ & $p$ & $\sigma_{\mathrm{e}}$ \\
\hline $25 \mathrm{~m}$ & $0.90 \times 10^{-13}$ & 1.37 & 0.09 \\
$50 \mathrm{~m}$ & $1.53 \times 10^{-13}$ & 1.47 & 0.13 \\
$75 \mathrm{~m}$ & $1.59 \times 10^{-13}$ & 1.48 & 0.11 \\
$100 \mathrm{~m}$ & $1.56 \times 10^{-13}$ & 1.48 & 0.10 \\
\hline
\end{tabular}

Table 3. Fitting parameters $A$ and $p$, for various vertical resolutions, corresponding to $C_{N_{\text {mean }}}^{2}$ values in the boundary layer.

\begin{tabular}{cccc}
\hline \hline$\Delta h$ & $A$ & $p$ & $\sigma_{\mathrm{e}}$ \\
\hline $25 \mathrm{~m}$ & $0.74 \times 10^{-12}$ & 1.46 & 0.07 \\
$50 \mathrm{~m}$ & $1.15 \times 10^{-12}$ & 1.53 & 0.11 \\
$75 \mathrm{~m}$ & $1.18 \times 10^{-12}$ & 1.54 & 0.11 \\
$100 \mathrm{~m}$ & $1.90 \times 10^{-12}$ & 1.60 & 0.08 \\
\hline
\end{tabular}

Table 4. The mean value and standard deviation of the ( $A$ and $p$ ) parameters, corresponding to $C_{N}^{2}$ dot chart.

\begin{tabular}{ccc}
\hline \hline $\mathrm{Y}$ & $<A> \pm \sigma_{A}$ & $<p> \pm \sigma_{p}$ \\
\hline$C_{N_{\text {median }}}^{2}$ & $(1.39 \pm 0.28) \times 10^{-13}$ & $1.45 \pm 0.04$ \\
$C_{N_{\text {mean }}}^{2}$ & $(1.24 \pm 0.42) \times 10^{-12}$ & $1.53 \pm 0.05$ \\
\hline
\end{tabular}

In Tables 2 and 3 are given the result of the fits between experimental and modeled data, for $C_{N_{\text {median }}}^{2}$ and $C_{N_{\text {mean }}}^{2}$, and for various vertical resolutions.

As it seems that there is no unique $\Delta h$ giving the best fit, we average the $A$ and $p$ values of Tables 2 and 3 , leading to Table 4.

Thus, the fitting functions of $C_{N}^{2}$ in the boundary layer are described as:

$$
C_{N_{\text {median }}}^{2}(h)=1.39 \times 10^{-13} h^{-1.45}
$$

and

$C_{N_{\text {mean }}}^{2}(h)=1.24 \times 10^{-12} h^{-1.53}$. 

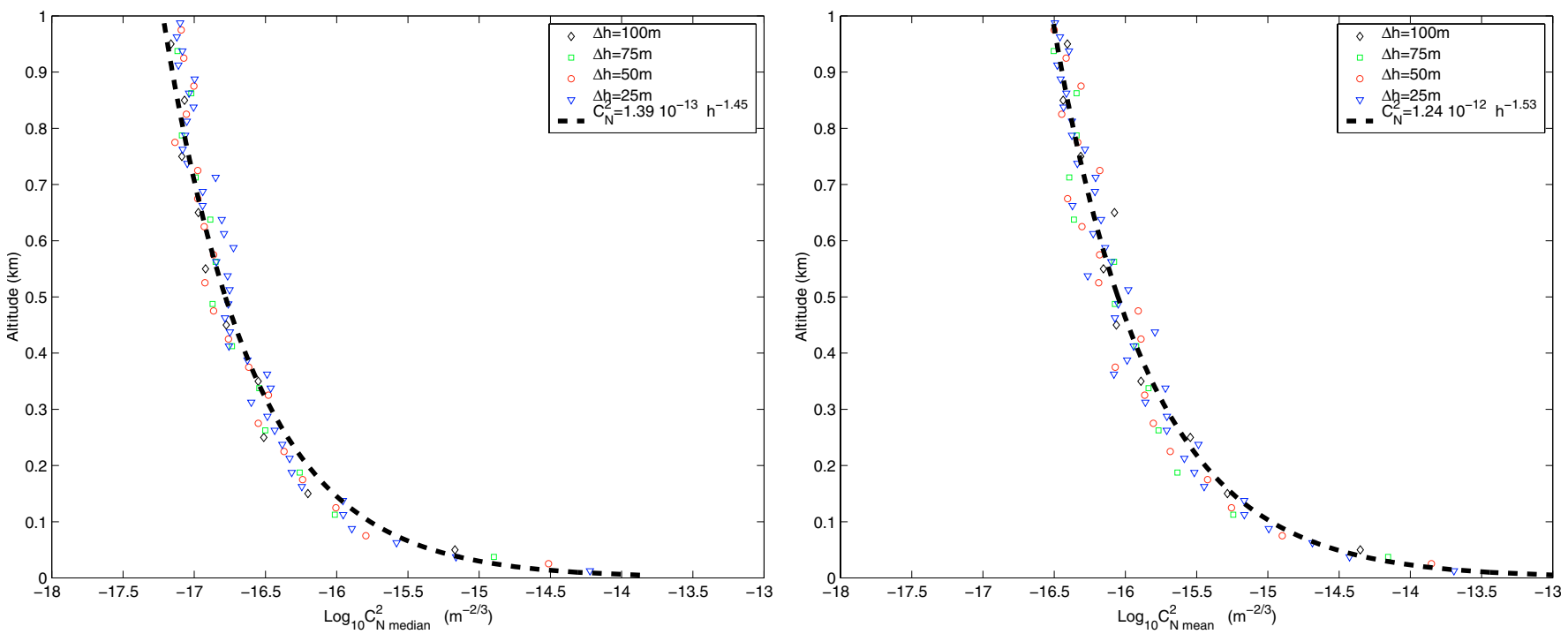

Fig. 2. $C_{N}^{2}$ vertical profile calculated for different layer thickness $\Delta h$, in the boundary layer. Right: mean profile; left: median profile; dotted-fat line: fitting function.

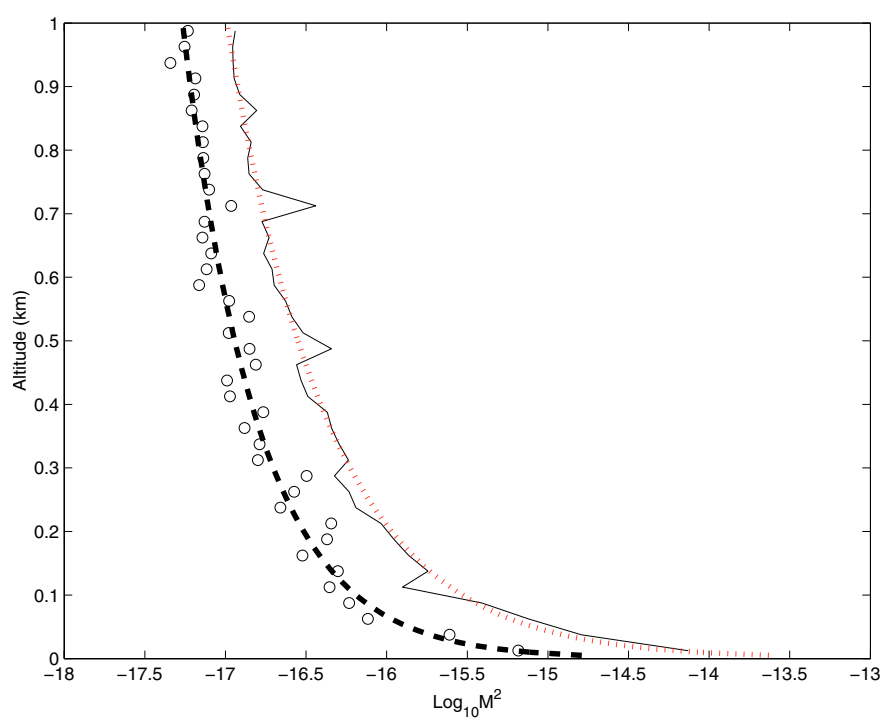

Fig. 3. $M^{2}$ vertical profile from sounding data computed for $\Delta h=$ $25 \mathrm{~m}$ in the boundary layer. o: median; solid line: mean; dotted line and dotted-fat line: functions obtained by performing a least squares exponential fit.

These functions show a height dependency close to $h^{-\frac{4}{3}}$ given by Wyngaard et al. (1971) but are in worse agreement with Gur'yanov $(1984,1988)$, who found an $h^{-2}$ dependency.

\section{- Vertical gradient of refractive index $M^{2}$ :}

$M^{2}$ statistical results in the boundary layer are presented in Figs. 3 and 4 and in Tables 5 and 6.

As for $C_{N}^{2}$, we averaged $A$ and $p$ values for $M^{2}$ and summarized them in Table 7 .

Thus, the fitting functions of $M^{2}$ in the boundary layer are described as:

$$
M_{\text {median }}^{2}(h)=1.22 \times 10^{-14} h^{-1.11}
$$

Table 5. Fitting parameters $A$ and $p$, for various vertical resolutions, corresponding to $M_{\text {median }}^{2}$ values in the boundary layer.

\begin{tabular}{cccc}
\hline \hline$\Delta h$ & $A$ & $p$ & $\sigma_{\mathrm{e}}$ \\
\hline $25 \mathrm{~m}$ & $0.92 \times 10^{-14}$ & 1.08 & 0.09 \\
$50 \mathrm{~m}$ & $1.09 \times 10^{-14}$ & 1.10 & 0.13 \\
$75 \mathrm{~m}$ & $1.25 \times 10^{-14}$ & 1.11 & 0.09 \\
$100 \mathrm{~m}$ & $1.64 \times 10^{-14}$ & 1.17 & 0.07 \\
\hline
\end{tabular}

Table 6. Fitting parameters $A$ and $p$, for various vertical resolutions, corresponding to $M_{\text {mean }}^{2}$ values in the boundary layer.

\begin{tabular}{cccc}
\hline \hline$\Delta h$ & $A$ & $p$ & $\sigma_{\mathrm{e}}$ \\
\hline $25 \mathrm{~m}$ & $2.45 \times 10^{-13}$ & 1.46 & 0.09 \\
$50 \mathrm{~m}$ & $1.45 \times 10^{-13}$ & 1.38 & 0.12 \\
$75 \mathrm{~m}$ & $1.45 \times 10^{-13}$ & 1.40 & 0.08 \\
$100 \mathrm{~m}$ & $1.69 \times 10^{-13}$ & 1.41 & 0.13 \\
\hline
\end{tabular}

Table 7. Mean and standard deviation of the $A$ and $p$ parameters, corresponding to median and mean $M^{2}$ values.

\begin{tabular}{ccc}
\hline \hline$Y$ & $<A> \pm \sigma_{A}$ & $<p> \pm \sigma_{p}$ \\
\hline$M_{\text {median }}^{2}$ & $(1.22 \pm 0.27) \times 10^{-14}$ & $1.11 \pm 0.04$ \\
$M_{\text {mean }}^{2}$ & $(1.76 \pm 0.41) \times 10^{-13}$ & $1.41 \pm 0.03$ \\
\hline
\end{tabular}

and

$M_{\text {mean }}^{2}(h)=1.76 \times 10^{-13} h^{-1.41}$.

\section{- Outer scale of turbulence $L_{0}$ :}

The results corresponding to the outer scale $L_{0}$ are illustrated in Figs. 5 and 6 and are summarized in Tables 8 and 9. $L_{\mathrm{o}}$ steeply decreases from ground level up to $200 \mathrm{~m}$, and then is almost constant between $200 \mathrm{~m}$ and $1 \mathrm{~km}$. 

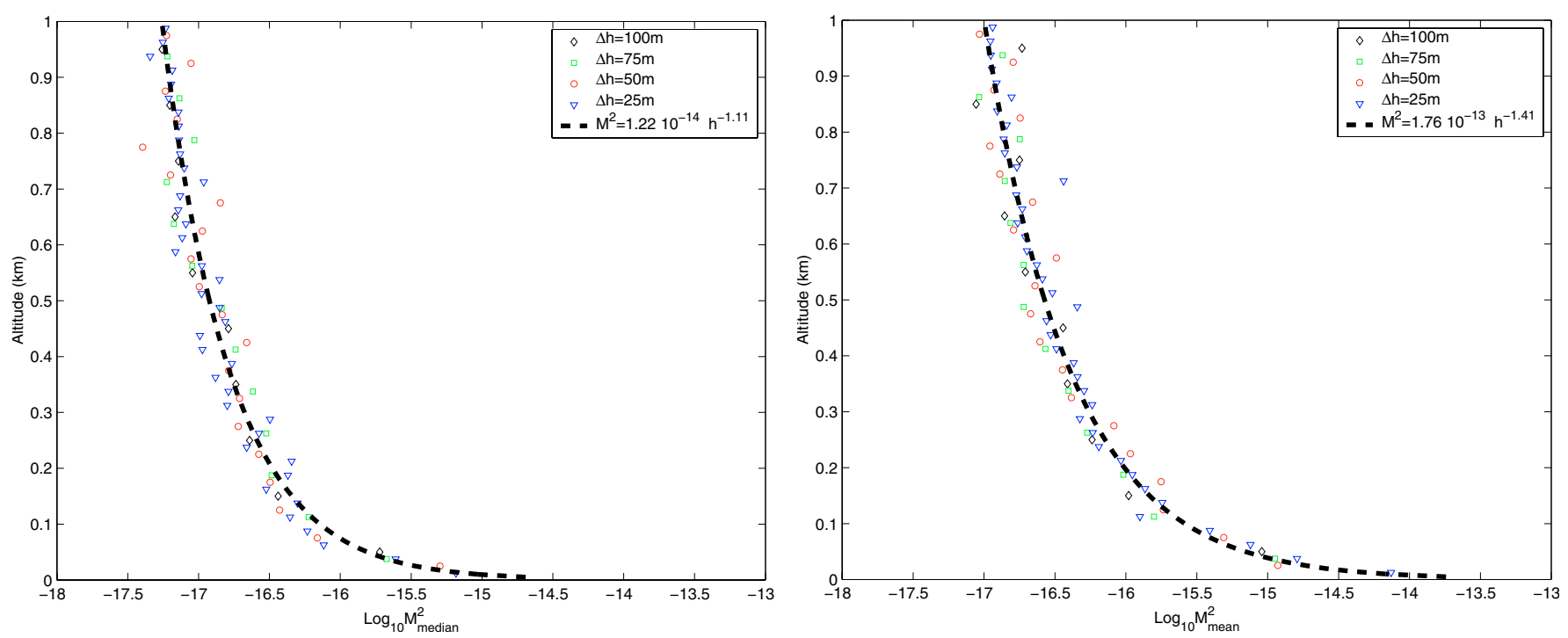

Fig. 4. $M^{2}$ vertical profile calculated for different layer thickness $\Delta h$, in the boundary layer. Right: mean profile; left: median profile; dotted-fat line: fitting function.

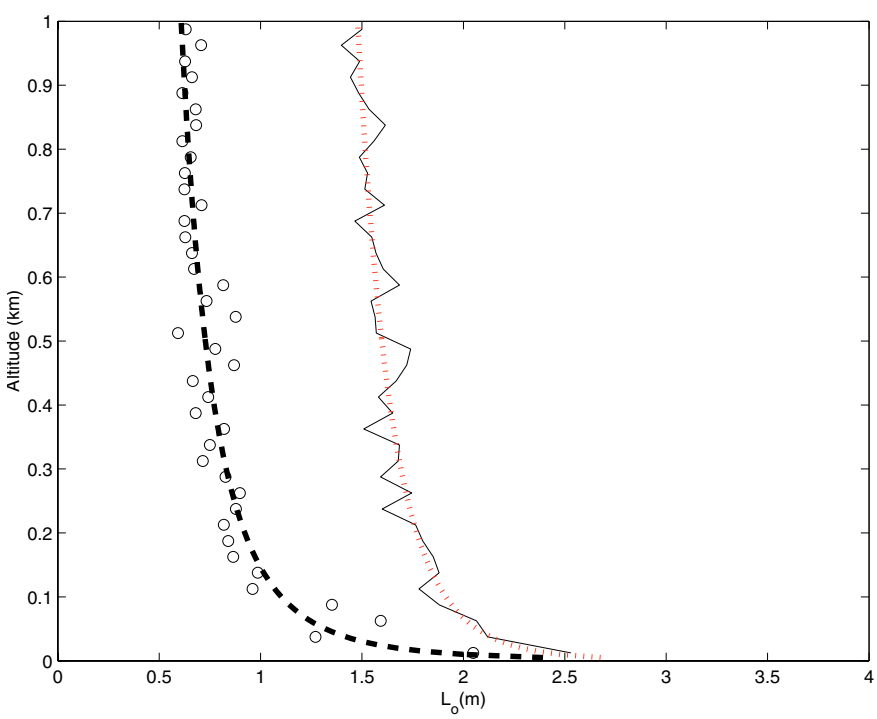

Fig. 5. $L_{\mathrm{o}}$ vertical profile from sounding data computed for $\Delta h=25 \mathrm{~m}$ in the boundary layer. o: median; solid line: mean; dotted line and dotted-fat line: functions obtained by performing a least squares exponential fit.

For $C_{N}^{2}$ and $M^{2}$, we averaged $A$ and $p$ values for the outer scale and summarized them in Table 10.

Fitting functions for $L_{\mathrm{o}}$ in the boundary layer are described as:

$L_{\mathrm{O}_{\text {median }}}(h)=3.78 h^{-0.27}$

and

$L_{\mathrm{O}_{\text {mean }}}(h)=3.21 h^{-0.11}$.

These functions disagree with $L_{\mathrm{o}}(h)=2 h^{-1 / 2}$ given by Good et al. (1988).
Table 8. Fitting parameters $A$ and $p$, for various vertical resolutions, corresponding to $L_{\mathrm{O}_{\text {median }}}$ values in the boundary layer.

\begin{tabular}{cccc}
\hline \hline$\Delta h$ & $A$ & $p$ & $\sigma_{\mathrm{e}}$ \\
\hline $25 \mathrm{~m}$ & 3.62 & 0.26 & 0.04 \\
$50 \mathrm{~m}$ & 3.64 & 0.26 & 0.03 \\
$75 \mathrm{~m}$ & 3.90 & 0.26 & 0.01 \\
$100 \mathrm{~m}$ & 3.98 & 0.27 & 0.02 \\
\hline
\end{tabular}

Table 9. Fitting parameters $A$ and $p$, for various vertical resolutions, corresponding to $L_{\mathrm{O}_{\text {mean }}}$ values in the boundary layer.

\begin{tabular}{cccc}
\hline \hline$\Delta h$ & $A$ & $p$ & $\sigma_{\mathrm{e}}$ \\
\hline $25 \mathrm{~m}$ & 3.20 & 0.11 & 0.01 \\
$50 \mathrm{~m}$ & 3.13 & 0.11 & 0.01 \\
$75 \mathrm{~m}$ & 3.20 & 0.11 & 0.01 \\
$100 \mathrm{~m}$ & 3.32 & 0.12 & 0.01 \\
\hline
\end{tabular}

\subsection{Free atmosphere}

All the 168 flights were processed with a $500 \mathrm{~m}$ large sliding window in order to compute $C_{N}^{2}, M^{2}$ and $L_{\mathrm{o}}$.

\section{- Structure constant of refractive index $C_{N}^{2}$ :}

Here we chose to compare our results to those of the Hufnagel (1974) model. This model has been developed by collecting the available experimental $C_{N}^{2}$ profiles and then working out a physically reasonable mathematical process which agrees with these profiles. Hufnagel synthetized a model valid for (ground $+3 \mathrm{~km})<h<24 \mathrm{~km}$ :

$$
\begin{aligned}
C_{N}^{2}=\{ & {\left[\left(2.2 \times 10^{-53}\right) h^{10}\left(\frac{W}{27}\right)^{2}\right] \exp \left(-\frac{h}{1000}\right) } \\
& \left.+\left(10^{-16}\right) \exp \left(-\frac{h}{1500}\right)\right\} \exp [r(h, t)]
\end{aligned}
$$



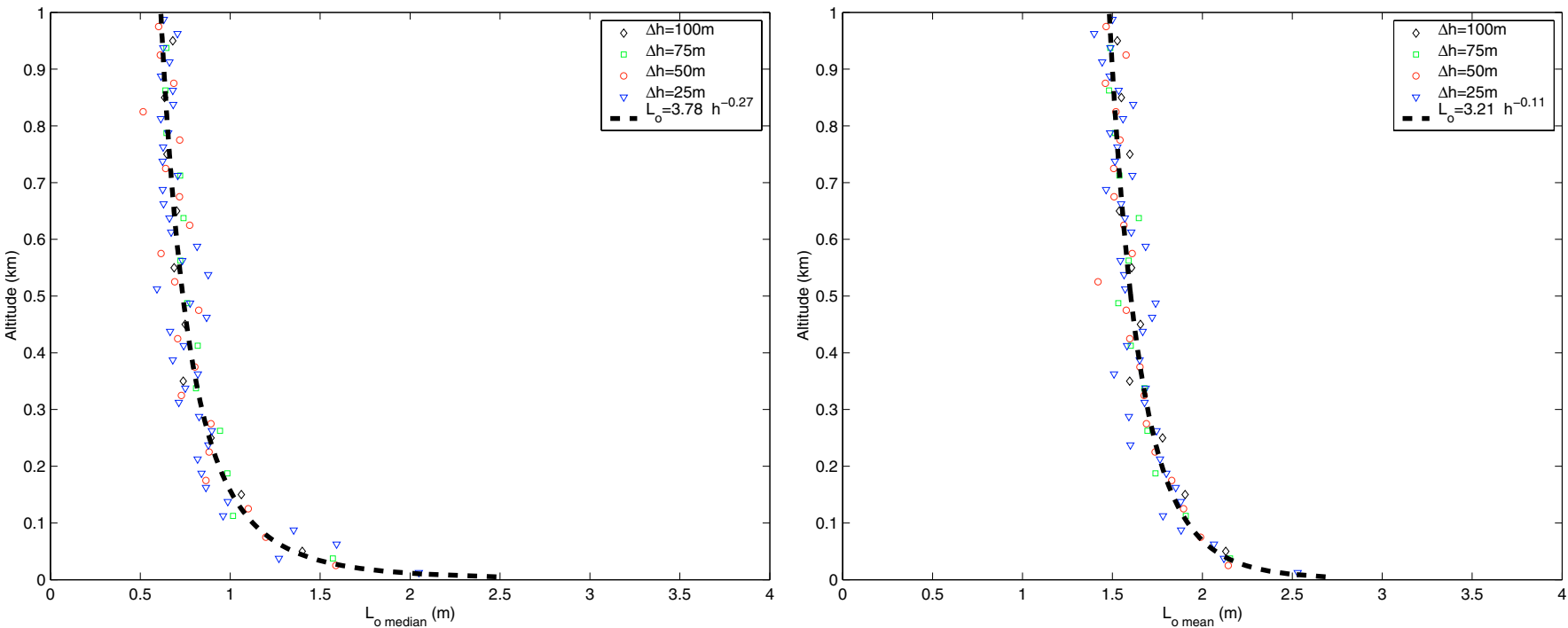

Fig. 6. $L_{\mathrm{o}}$ vertical profile calculated for different layer thickness $\Delta h$, in the boundary layer. Right: mean profile; left: median profile; dotted-fat line: fitting function.

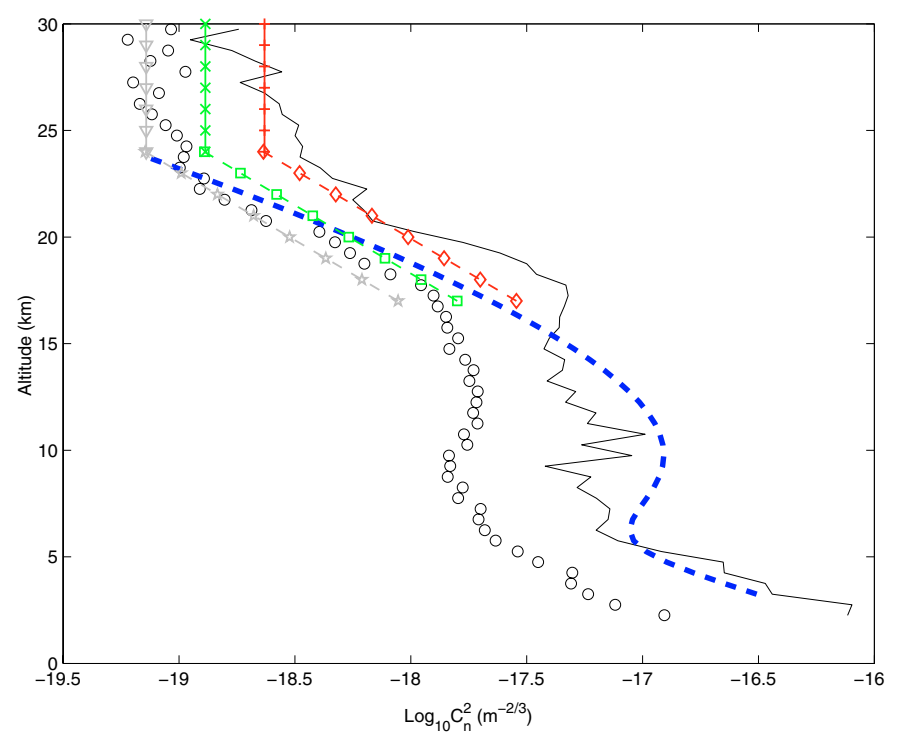

Fig. 7. Comparison of $C_{N}^{2}$ vertical profile deduced from sounding data and those of the Brown-Beland (BB) model. o: median; solid line: mean; Dotted-fat line: Hufnagel model; $\mathrm{x}: C_{N}^{2}=1.310^{-19} \mathrm{BB} 1$ model valid for $24 \mathrm{~km}<h<30 \mathrm{~km}$; +: BB1 model multiplied by 1.8 ; triangles: BB1 model divided by 1.8 ; squares: $C_{N}^{2}(h)=7.1 \times 10^{-16} \mathrm{e}^{-0.359 h}$ BB2 model valid for $17 \mathrm{~km}<h<24 \mathrm{~km}$; diamond: BB2 model multiplied by 1.8 ; pentagram: BB2 divided by 1.8 .

where $h$ is the altitude in meters above sea level and $r$ is a Gaussian random variable such as $<\exp (r)>=e=2.7 \times$ $W\left(\mathrm{~m} \mathrm{~s}^{-1}\right)$ is a correlation factor related to scintillation, expressed by:

$W=\left[\frac{1}{15 \mathrm{~km}} \int_{5 \mathrm{~km}}^{20 \mathrm{~km}} V^{2}(h) \mathrm{d} h\right]^{\frac{1}{2}}$

and $V(h)$ is the wind speed at altitude $h$.

From our 168 soundings, we obtained $W=18.6 \mathrm{~m} \mathrm{~s}^{-1}$, which is very close to the $18 \mathrm{~m} \mathrm{~s}^{-1}$ found by Hufnagel.
Table 10. Mean and standard deviation of the $A$ and $p$ parameters, corresponding to median and mean $L_{\mathrm{o}}$ values.

\begin{tabular}{ccc}
\hline \hline$Y$ & $<A> \pm \sigma_{A}$ & $<p> \pm \sigma_{p}$ \\
\hline$L_{\mathrm{O}_{\text {median }}}$ & $3.78 \pm 0.15$ & $0.27 \pm 0.005$ \\
$L_{\mathrm{O}_{\text {mean }}}$ & $3.21 \pm 0.07$ & $0.11 \pm 0.002$ \\
\hline
\end{tabular}

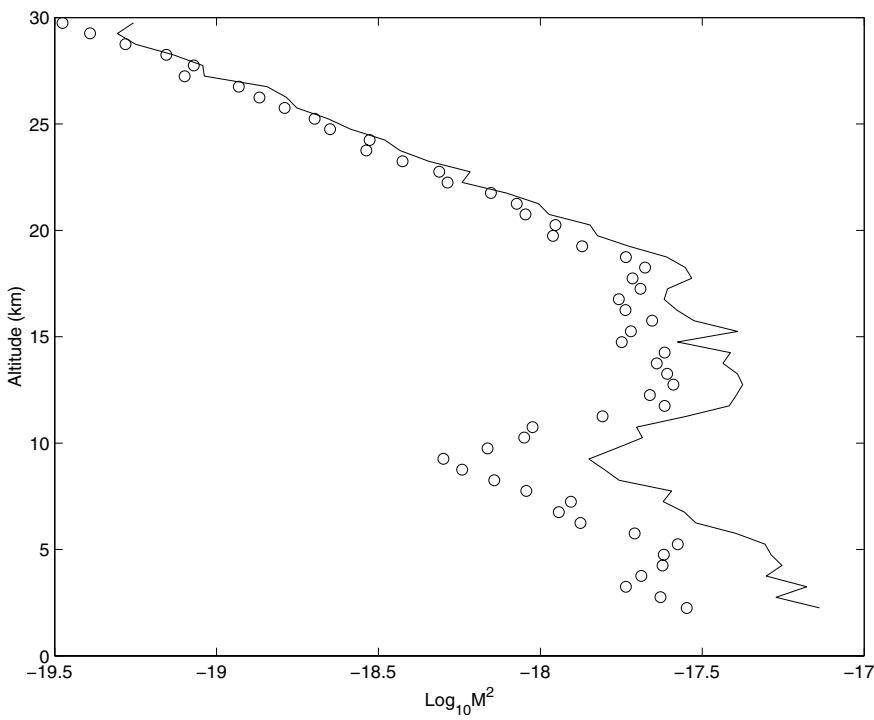

Fig. 8. $M^{2}$ vertical profile computed from sounding data in the free atmosphere. o: median; solid line: mean.

In Fig. 7, one can see a good agreement between the $C_{N}^{2}$ mean Hufnagel profile and ours. Beyond $24 \mathrm{~km}$, the $C_{N}^{2}$ decreases very slowly. This trend agrees well with that obtained by Brown \& Beland (1988):

$C_{N}^{2}(h)=7.1 \times 10^{-16} \mathrm{e}^{-0.359 h}(\times / \div$ by 1.8$)$

valid for $17 \mathrm{~km} \leq h \leq 24 \mathrm{~km}$, with $h$ in $\mathrm{km}$. 


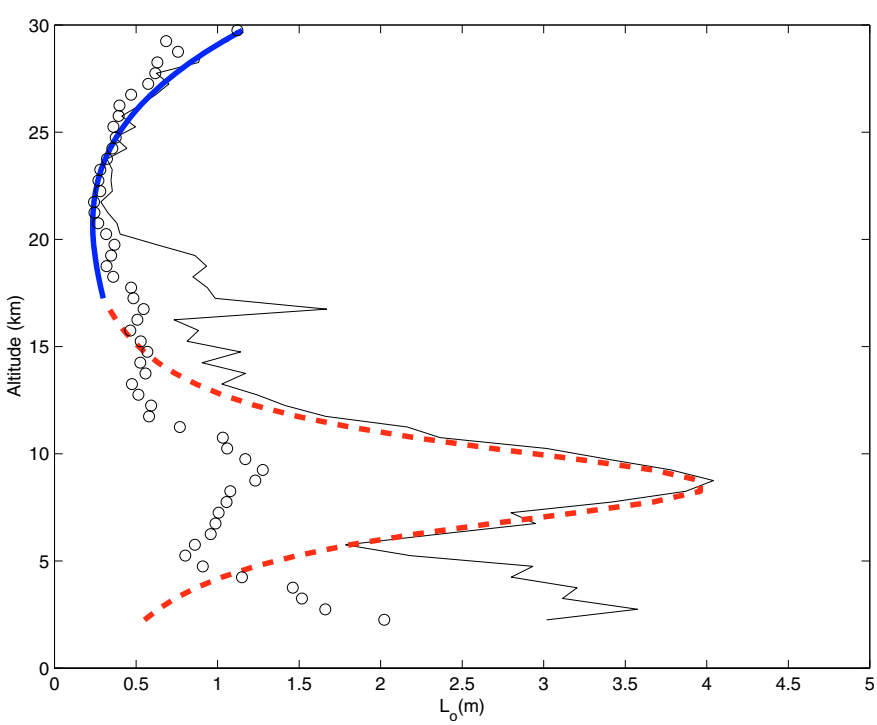

Fig. 9. Comparison of $L_{\mathrm{o}}$ vertical profil deduced from our soundings data and the result of Brown-Beland model (solid fat line) and Coulman-Vernin model (dotted fat line). o: median, solid line: mean.

\section{- Vertical gradient of refractive index $M^{2}$ :}

Figure 8 shows the $M^{2}$ results in the free atmosphere. We notice that the $M^{2}$ profile follows an almost linear variation with three different slopes.

\section{- Outer scale of turbulence $L_{0}$ :}

In Fig. 9, our $L_{\mathrm{o}}$ profiles are superimposed on that given by the Beland-Brown (1988) and Coulman-Vernin (1988) models. The Beland-Brown model is approximated by:

$$
\begin{aligned}
L_{\mathrm{o}}(h)= & (0.307)+(-0.0324)(h-17) \\
& +(0.00167)(h-17)^{2}+(0.000476)(h-17)^{3}
\end{aligned}
$$

valid for $17 \mathrm{~km} \leq h \leq 30 \mathrm{~km}$, with $\mathrm{h}$ in $\mathrm{km}$. the CoulmanVernin model is fitted with:

$$
L_{\mathrm{o}}(h)=\frac{4}{1+\left(\frac{h-8500}{2500}\right)^{2}}
$$

valid for $2 \mathrm{~km}<h<17 \mathrm{~km}$.

One can see a spectacular agreement between our $L_{\mathrm{o}}$ mean profile and the Coulman-Vernin model in the $5 \mathrm{~km}<h<17 \mathrm{~km}$ range, and between our $L_{\mathrm{o}}$ mean profile and Beland-Brown model in the $20 \mathrm{~km}<h<30 \mathrm{~km}$ range. As the SCIDAR, in this epoch, did not detect the turbulent layers in the first kilometer, the model Coulman-Vernin model could not evaluate the outer scale of turbulence at low altitude. Accordingly, in the low layers, our measurements fill the gap in the Coulman-Vernin model.

\subsection{Wavefront outer scale $\left(£_{0}\right)$}

Generally, The size of the largest eddies in each layer gives a rather good approximation of the outer scale $L_{0}$. Star light crosses several turbulent layers, each one is characterized by a

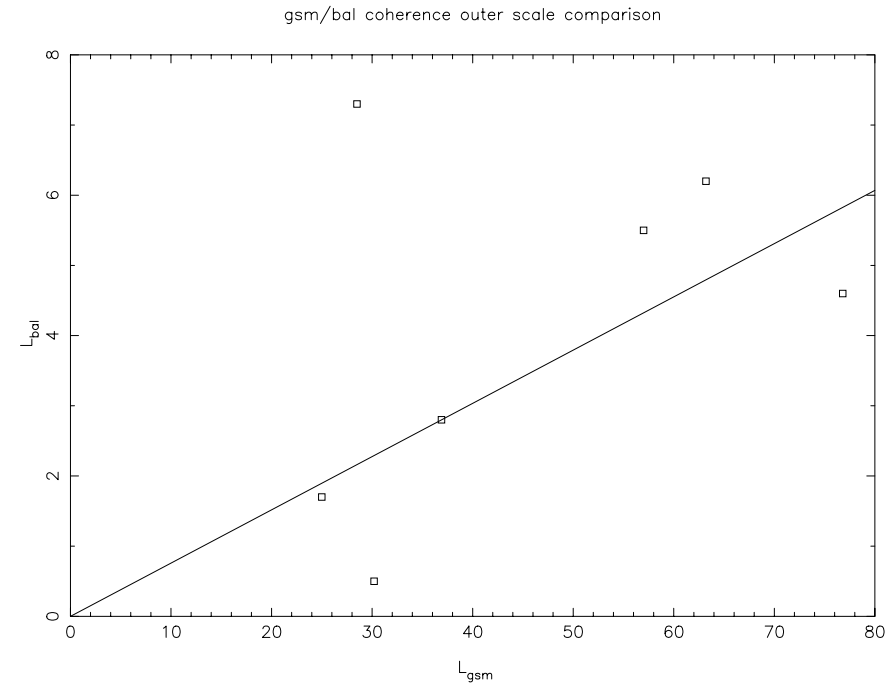

Fig. 10. Comparison of the coherence outer scale $£$ deduced from GSM and balloons during Gemini campaign, at Cerro Pachón, from 2nd to 9th of October 1998.

turbulence intensity $C_{N}^{2}$ and a local outer scale $L_{\mathrm{o}}$. The combination of all these effects on the wavefront passing through the whole atmosphere results in defining a coherence wavefront outer scale $£_{0}$ (Borgnino 1990), which is connected to the local geophysical parameter $\left(L_{\mathrm{o}}\right)$ and the optical energy of turbulence $C_{N}^{2}$ :

$\mathfrak{f}_{\mathrm{o}}^{-1 / 3}=\frac{\int_{\mathrm{o}}^{+\infty} L_{\mathrm{o}}^{-1 / 3}(h) C_{N}^{2}(h) \mathrm{d} h}{\int_{\mathrm{o}}^{+\infty} C_{N}^{2}(h) \mathrm{d} h}$.

Several methods were worked out to measure $£_{0}$. One will find a summary of most of the wave-front outer scale measurements in Avila et al. (1997). Generally they are based on interferometric techniques or wavefront analysis. Mariotti (1984) have used the I2T interferometer of CERGA to estimate the outer scales of the order of $8 \mathrm{~m}$. On the other hand, by using the Mark III interferometer (at Mount-Wilson), Colavita et al. (1987) estimated the outer scales higher than $2 \mathrm{~km}$. Buscher et al. (1991) found values of about $30 \mathrm{~m}$ with the same interferometer, invalidating the Colavita result.

The angle of arrival fluctuations acquired from a HartmannShack analyzer's made possible to find outer scales between $5 \mathrm{~m}$ and $8 \mathrm{~m}$ (Tallon 1989). In the framework of the COME ON experiment Rigaut et al. (1991), and Rousset et al. (1991) found an outer scale of about $50 \mathrm{~m}$.

The analysis of the DIMM data show that the outer scale can take a value between $5 \mathrm{~m}$ and $100 \mathrm{~m}$ (Ziad et al. 1994). In addition, the first results of $£_{0}$ monitoring with the Grating Scale Monitor at Mont-Gros (Nice) and the Calern plateau respectively show $£_{\mathrm{o}}$ values of the order of $50 \mathrm{~m}$ and $300 \mathrm{~m}$. All the previous methods are model-dependent, which means that experimental data are analysed through the assumption of a Kolmogorov spectrum, but truncated at its extremities in different ways (see Lutomirski \& Yura 1971; Gardner 1976, and references therein).

On the other hand, Fuchs (1995) and Coulman et al. (1988) estimated the outer scale from Eq. (2) where $L_{\mathrm{o}}$ is defined 
as an integral length, as it is assumed in this article. All our $L_{\mathrm{o}}$ measurements are less than $5 \mathrm{~m}$. Such a large difference is likely due to the different definitions of the outer scale. Fortunately, during the Gemini campaign in 1998, we had both the GSM and the instrumented balloons working simultaneously. In Fig. 10 we give $7 £_{0}$ measurements deduced from the GSM and the balloons, taken during the Cerro Pachón site testing campaign, in October 1998. If we exclude one measurement $\left(£_{\mathrm{gsm}}=28.5 \mathrm{~m}, \mathfrak{b}_{\mathrm{bal}}=7.3 \mathrm{~m}\right)$ a linear regression gives a line $\mathfrak{f}_{\mathrm{bal}}=0.076 \mathfrak{g}_{\mathrm{gsm}}$. This result is not an explanation, but encourages us to analyse in more detail the influence of the various definitions of the outer scale, depending upon the type of technique used.

From our 168 soundings and using Eq. (21) we found $£_{\text {bal median }}=1.27 \mathrm{~m}$ and $£_{\text {bal mean }}=3.5 \mathrm{~m}$. According to our previous discussion, this would lead to a model-dependent $£_{\text {model median }}=16.7 \mathrm{~m}$ and $£_{\text {model mean }}=46 \mathrm{~m}$, which is very close to the values issued by the GSM (Avila et al. 1997).

\section{Conclusion}

The analysis of radiosonde data performed at various different astronomical sites allows us to determine the mean and median profiles of $C_{N}^{2}, M^{2}$ and $L_{\mathrm{o}}$ in the boundary layer and the free atmosphere separately.

A conclusion to be drawn from this work is the decreasing power law with altitude for each of these parameters. The deduced models are based on a statistical approach and lead to a better understanding of the behavior of these parameters relevant to the optical quality of the atmosphere in the boundary layer and in the free atmosphere. Our parametrization fills certain gaps found in other models such as the Coulman-Vernin model for $L_{\mathrm{o}}$ or Hufnagel model for $C_{N}^{2}$, giving the statistical behavior of these parametrers from the ground up to $30 \mathrm{~km}$.

An other important result obtained in the boundary layer is the $h^{-\frac{4}{3}}$ dependency of $C_{N}^{2}$ with height, which confirms Wyngaard et al. (1971) result during the night, but invalidates the $h^{-2}$ Gur' yanov (1984) dependency.

In the free atmosphere, the Hufnagel model agrees well with our results. In the same way, the comparison with the Brown-Beland models is spectacular for the $L_{\mathrm{o}}$ trend in the stratosphere. In the $5-10 \mathrm{~km}$ region there is also an impressive agreement between the Coulman-Vernin model and our's.

It is the first time that a complete median and average $C_{N}^{2}(h), M^{2}(h)$ and $L_{\mathrm{o}}(h)$ profile, from the ground up to $30 \mathrm{~km}$, is available. Unlike other previous attempts, our results cover a wide variety of latitudes and longitudes, increasing their validity.

\section{References}

Avila, R., Ziad, A., Borgnino, J., Martin, F., \& Agabi, A. 1997, J. Opt. Soc. Am., 14, 3070

Barletti, R., Ceppatelli, G., Moroder, E., Paterno, L., \& Righini, A. 1974, J. Geophys. Res., 79, 4545

Barletti, R., Ceppatelli, G., Paterno, L., Righini, A., \& Speroni, N. 1977, A\&A, 54, 649

Beland, R. R., \& Brown, J. H. 1988, Phys. Scr., 37, 419

Borgnino, J. 1990, Appl. Opt., 29, 1863

Bougeault, P., De Hui, C., Fleury, B., \& Laurent, J. 1995, Appl. Opt., 34,3481

Brown, J. H., \& Beland, R. R. 1988, Phys. Scr., 37, 424

Bufton, J. L. 1973a, J. Atmos. Sci., 30, 83

Bufton, J. L. 1973b, Appl. Opt., 12, 1785

Colavita, M. M., Shao, M., \& Staelin, D. H. 1987, Appl. Opt., 26, 4106

Buscher, D. F., Armstrong, J. T., Mozurkewich, D., et al. 1991, ESO Proc., Garching, 403

Coulman, C. E. 1973, Boundary-Layer Meteor., 4, 196

Coulman, C. E., Vernin, J., Coqueugniot, Y., \& Caccia, J. L. 1988, Appl. Opt., 27, 155

Fried, D. L. 1966, J. Opt. Soc. Am., 56, 1372

Fuchs, A. 1995, Thèse de doctorat, Université de Nice, Nice, France

Gardner, C. S. 1976, Appl. Opt., 15, 2539

Good, R. E., Beland, R. R., Murphy, E. A., Brown, J. H., \& Dewan, E. M. 1988, SPIE, 928, 165

Gur'yanov, A. E. 1984, Sov. Astron., 28, 343

Gur'yanov, A. E., Irkaev, B. N., Kallistratova, M. A., et al. 1988, Sov. Astron., 32, 328

Hufnagel, R. E. 1974, O.S.A. topical meeting on optical propagation through turbulence, July 9-11, Boulder, Colorado

Lutomirski, R., \& Yura, H. 1971, J. Opt. Soc. Am., 61, 482

Mariotti, J. M., \& Di Benedetto, G. P. 1984, Proc. of the IAU Coll. Garching, 79, 257

Masciadri, E. 1998, Thèse de doctorat, Université de Nice, Nice, France

Rigaut, F., Rousset, G., Kern, P., et al. 1991, A\&A, 250, 280

Roddier, F. 1981, in Progress in Optics, ed. E. Wolf, 19, 281

Roddier, F., Cowie, L., Graves, J. E., et al. 1990, SPIE, 1236, 485

Rousset, G., Madec, P. Y., \& Rigaut, F. 1991, Digest Topical Meeting on Atmospheric Volume and Surface Scattering and Propagation, Florence, 77

Sarazin, M. 1987, ESO-VLT working group on site evaluation, VLT report 55

Tallon, M. 1989, Thèse de Doctorat, Université de Nice, Nice, France

Tatarski, V. I. 1961, Wave propagation in random media (Dover, New York)

Vernin, J., \& Muñoz-Tuñon, C. 1992, A\&A, 257, 811

Wyngaard, J. C., Izumi, Y., \& Collins, S. A. 1971, J. Opt. Soc. Am., 61,1646

Ziad, A., Borgnino, J., Martin, F., \& Agabi, A. 1994, A\&A, 282, 1021 